\title{
Plasminogen activator inhibitor-1 deficiency enhances subchondral osteopenia after induction of osteoarthritis in mice
}

\author{
Akihiro Moritake ${ }^{1,2}$, Naoyuki Kawao ${ }^{2}$, Kiyotaka Okada ${ }^{2}$,Kohei Tatsumi ${ }^{2}$, Masayoshi Ishida², Katsumi Okumoto ${ }^{3}$, \\ Osamu Matsuo ${ }^{2}$, Masao Akagi ${ }^{1}$ and Hiroshi Kaji ${ }^{2 *}$
}

\begin{abstract}
Background: Subchondral osteopenia is important for the pathophysiology of osteoarthritis (OA). Although previous studies suggest that plasminogen activator inhibitor-1 (PAl-1), an inhibitor of fibrinolysis, is related to bone metabolism, its role in OA remains unknown. We therefore investigated the roles of PAl-1 in the subchondral bone in OA model mice.
\end{abstract}

Methods: Wild type (WT) and PAl-1-deficient (KO) mice were ovariectomized (OVX), and then destabilization of the medial meniscus (DMM) surgery was performed.

Results: DMM and OVX significantly decreased the trabecular bone mineral density of the subchondral bone evaluated by quantitative computed tomography in PAl-1 KO mice. The effects of OVX and/or PAl-1 deficiency on the OARSI score for the evaluation of the progression of knee degeneration were not significant. PAI-1 deficiency significantly augmented receptor activator nuclear factor KB ligand mRNA levels enhanced by IL-1 $\beta$ in mouse primary osteoblasts, although it did not affect osteoblast differentiation. Moreover, PAl-1 deficiency significantly increased osteoclast formation from mouse bone marrow cells.

Conclusion: We showed that PAl-1 deficiency accelerates the subchondral osteopenia after induction of OA in mice. PAl1 might suppress an enhancement of bone resorption and subsequent subchondral osteopenia after induction of OA in mice.

Keywords: Osteoarthritis, Osteoclast, Ovariectomy, Plasminogen activator inhibitor-1, Subchondral bone

\section{Background}

Osteoarthritis (OA) is one of the most frequent diseases in orthopedic clinics. Despite being a disease with a major impact on the medical economy, OA has been generally recognized as a disease that occurs with aging, and its treatment has been only limited to artificial joint replacement when the disease progresses to a severe stage. Although OA has been mainly considered to be due to the wear of cartilage from mechanical stress [1], the prevalence of OA has been reported to be increased in postmenopausal women [2-4]. Moreover, a previous

\footnotetext{
* Correspondence: hkaji@med.kindai.ac.jp

${ }^{2}$ Department of Physiology and Regenerative Medicine, Kindai University Faculty of Medicine, 377-2 Ohnohigashi, Osakasayama, Osaka 589-8511, Japan

Full list of author information is available at the end of the article
}

study suggested that osteoporosis aggravates cartilage damage in knee OA model rats [5]. These findings suggest the possibility that bone metabolism and osteoporosis affects the progression of arthropathy.

Changes in bone around the joint, especially the subchondral bone, have recently been suggested to be involved in the disease condition from a relatively early stage in OA. Particularly, the subchondral bone is influenced by a high bone turnover state. Radin et al. suggested that the integrity of the articular cartilage depends on the underlying bone strength [6]. They speculated that cartilage degeneration is prone to progress by load stress in the osteoporotic state of the subchondral bone. Accelerated bone turnover by osteoblasts and osteoclasts is indispensable for remodeling of the subchondral bone in OA [7]. Moreover, a recent study 
indicated that the promotion of subchondral bone turnover is accompanied by specific structural changes in the subchondral trabecular bone of the OA joint [8]. These findings suggest that maintenance of bone mass in the subchondral bone may be important for the treatment of arthropathy $[9,10]$. However, the details of the mechanisms of subchondral osteopenia after induction of OA and the role of estrogen deficiency remain unclear.

Plasminogen activator inhibitor-1 (PAI-1) is a serine protease inhibitor that primarily inhibits tissue- and urokinase-type plasminogen activators, and acts as an inhibitor of fibrinolysis [11, 12]. PAI-1 possesses a fibrinolysis-independent function in various tissues [12]. PAI-1 is expressed in various cells, including bone and cartilage, as well as in the extracellular matrix. We previously revealed that PAI-1 is involved in osteopenia induced by diabetes and glucocorticoid excess as well as diabetes-induced delayed bone repair using PAI-1deficient mice [13-15]. However, the roles of PAI-1 in the pathogenesis of $\mathrm{OA}$ and osteoclastic bone resorption still remain unknown.

In the present study, we therefore investigated the roles of PAI-1 in the subchondral bone and cartilage changes in OA model mice using wild-type (WT) and PAI-1-deficient (KO) mice.

\section{Methods}

\section{Materials}

Macrophage colony-stimulating factor (M-CSF) and receptor activator of nuclear factor- $\mathrm{kB}$ ligand (RANKL) were purchased from Wako (Osaka, Japan). SB431542, interleukin-1 $\beta$ (IL-1 $\beta$ ) and transforming growth factor- $\beta_{1}$ (TGF- $\beta_{1}$ ) were obtained from Tocris (Bristol, UK), R\&D systems (Minneapolis, MN, USA) and Sigma (St Louis, MO, USA), respectively. Bone morphogenetic protein-2 (BMP-2) was obtained from Pfizer Inc. (Groton, CT, USA).

\section{Animals}

PAI-1 KO and counterpart WT mice were used in this study. WT and PAI-1 KO mice were kindly provided by D. Collen (University of Leuven, Leuven, Belgium) and were bred at Kindai University Faculty of Medicine animal facility. The genetic background of all mice was mixed C57BL/6 J (81.25\%) and 129/SvJ (18.75\%). Female WT and PAI-1 KO mice were randomly divided into four groups: WT/Sham $(n=11)$, WT/ovariectomy $(\mathrm{OVX})(\mathrm{n}=11)$, PAI-1 KO/Sham $(n=10)$ and PAI-1 KO/OVX ( $\mathrm{n}=11)$. Seven-week-old female WT and PAI$1 \mathrm{KO}$ mice were ovariectomized or sham-operated as previously described [16]. One week after the OVX or sham surgery, all mice received destabilization of the medial meniscus (DMM) surgery on the right knee due to induction of OA and sham surgery on the left knee. Eight weeks after the DMM surgery, these mice were subjected to analysis. Food and water were provided ad libitum. The room temperature was kept at $24 \pm 1{ }^{\circ} \mathrm{C}$ with a $12 \mathrm{~h}: 12 \mathrm{~h}$ light/dark cycle. All experiments were performed according to the guidelines of the National Institutes of Health and the institutional rules for the use and care of laboratory animals at Kindai University.

\section{DMM surgery}

The DMM model, which provided high reproducibility and a slower progression of $\mathrm{OA}$ in mice, was used for evaluation of changes in subchondral bone and cartilage in the OA state in WT and PAI-1 KO mice. DMM surgery was performed on the right knee of mice as previously described [17]. Briefly, under anesthesia induced by $2 \%$ isoflurane, the anterior skin was cut by $5 \mathrm{~mm}$ to expose the joint capsule in mice. To expose the medial meniscus, the joint capsule immediately medial to the patellar tendon was incised. After transection of the meniscotibial ligament, skin was closed sterilely. A sham surgery was performed on the left knee using the same procedure with no transection of the meniscotibial ligament.

\section{Quantitative computed tomography (qCT) analysis}

For qCT analysis of the bone mineral density (BMD), mice were anesthetized using $2 \%$ isoflurane and scanned using an experimental animal CT system (LaTheta LCT200, Hitachi Aloka Medical, Tokyo, Japan) [18]. CT scans were performed at a tube voltage of $50 \mathrm{kVp}$, a tube current of $500 \mu \mathrm{A}$, an integration time of $3.6 \mathrm{~ms}$, an axial field of view of $48 \mathrm{~mm}$, and an isotopic voxel size of $24 \mu \mathrm{m}$. To assess the trabecular BMD of the tibial subchondral bone, regions of interest (ROI) were defined as $240-\mu \mathrm{m}$ (10-slice) segments from $72 \mu \mathrm{m}$ proximal to the end of the proximal growth plate towards the joint. This ROI was $200 \mu \mathrm{m}$ distal towards the joint, compared to ROI for the assessment of the trabecular BMD for systemic osteopenia in tibia. An increase in bone mineral content occurs in the most proximate subchondral region of the cartilage tissues in the knee. We therefore employed the most proximate trabecular bone region with a decrease in BMD with DMM and OVX in our preliminary experiments as the ROI for the measurement of BMD of the subchondral bone. Bone parameters were analyzed using LaTheta software (version3.40). A threshold density of $160 \mathrm{mg} / \mathrm{cm}^{3}$ was set to distinguish mineralized from unmineralized tissue. The density range was calibrated daily with a phantom supplied by the manufacturer.

\section{Histology analysis}

Mice were euthanized with excess isoflurane 8 weeks after DMM surgery. The knee samples were removed, fixed in $4 \%$ paraformaldehyde for $24 \mathrm{~h}$, and decalcified in $22.5 \%$ formic acid and $340 \mathrm{mM}$ sodium citrate 
solution for $24 \mathrm{~h}$. After demineralization, specimens were embedded in paraffin and cut into $5-\mu \mathrm{m}$ sections. The sections were stained with hematoxylin/eosin and photographed under a microscope (BZ-9000, Keyence, Osaka, Japan). The sections were stained with Safranin$\mathrm{O}$ and fast green, and subsequently counterstained with hematoxylin. Each knee was evaluated for the progression of arthropathy with 6 grades using the Osteoarthritis Research Society International (OARSI) histological scoring system [19].

\section{Quantitative real-time PCR}

Total RNA was extracted from primary osteoblasts using the RNeasy Mini Kit (Qiagen, Tokyo, Japan). One $\mu \mathrm{g}$ total RNA was reverse transcribed using a HighCapacity cDNA Reverse Transcription Kit (Applied Biosystems, Foster, CA, USA). Quantitative real-time PCR was performed using StepOnePlus and the Fast SYBR Green PCR Master Mix (Life Technologies, Tokyo, Japan) as previously described [13]. A list of primers used is shown in Table 1. The specific mRNA amplification of the target was determined as the $\mathrm{Ct}$ value, which was followed by normalization by the glyceraldehyde 3phosphate dehydrogenase (GAPDH) mRNA level.

Table 1 Primers used for real-time PCR experiments

\begin{tabular}{|c|c|c|}
\hline Gene & & Primer sequence \\
\hline \multirow[t]{2}{*}{ PAl-1 } & Forward & 5'-TTCAGCCCTTGCTTGCCTC-3' \\
\hline & Reverse & 5'-ACACTITTACTCCGAAGTCGGT-3' \\
\hline \multirow[t]{2}{*}{ TGF- $\beta_{1}$} & Forward & 5'-CCTCTGTCACCTGCTCAACA-3' \\
\hline & Reverse & 5'-GATGAATTGGCGTGGAATCT-3' \\
\hline \multirow[t]{2}{*}{ Runx2 } & Forward & 5'-AAATGCCTCCGCTGTTATGAA-3' \\
\hline & Reverse & 5'-GCTCCGGCCCACAAATCT-3' \\
\hline \multirow[t]{2}{*}{ Osterix } & Forward & 5'-AGCGACCACTTGAGCAAACAT-3' \\
\hline & Reverse & 5'-GCGGCTGATTGGCTTCTTCT-3' \\
\hline \multirow[t]{2}{*}{ ALP } & Forward & 5'-ATCTTTGGTCTGGCTCCCATG-3' \\
\hline & Reverse & 5'-TTTCCCGTTCACCGTCCAC-3' \\
\hline \multirow[t]{2}{*}{ OCN } & Forward & 5'-CCTGAGTCTGACAAAGCCTTCA-3' \\
\hline & Reverse & 5'-GCCGGAGTCTGTTCACTACCTT-3' \\
\hline \multirow[t]{2}{*}{ RANKL } & Forward & 5'- CACAGCGCTTCTCAGGAGCT-3' \\
\hline & Reverse & 5'-CATCCAACCATGAGCCTTCC-3' \\
\hline \multirow[t]{2}{*}{ OPG } & Forward & 5'-AGTCCGTGAAGCAGGAGT-3' \\
\hline & Reverse & 5'-CCATCTGGACATITITTGCAAA-3' \\
\hline \multirow[t]{2}{*}{ GAPDH } & Forward & 5'-AGGTCGGTGTGAACGGATTTG-3' \\
\hline & Reverse & 5'-GGGGTCGTTGATGGCAACA-3' \\
\hline
\end{tabular}

PAl-1 plasminogen activator inhibitor-1, TGF- $\beta_{1}$ transforming growth factor- $\beta 1$, ALP alkaline phosphatase, OCN osteocalcin RANKL receptor activator of nuclear factor-KB ligand, OPG osteoprotegerin, GAPDH glyceraldehyde-3-phosphate dehydrogenase
Primary osteoblasts and bone marrow stromal cells Primary osteoblastic cells were obtained from the calvaria of 3-day-old female WT and PAI-1 KO mice, as previously described [20]. The calvaria was removed from soft tissue, and digested 4 times with $1 \mathrm{mg} / \mathrm{mL}$ collagenase and $0.25 \%$ trypsin for $20 \mathrm{~min}$ at $37{ }^{\circ} \mathrm{C}$. Cells from second, third and fourth digestions were collected and grown in Minimum Essential Medium Alpha Modification ( $\alpha$-MEM; Wako) with 10\% fetal bovine serum (FBS; HyClone, Logan, UT, USA). The medium was changed twice a week.

Bone marrow stromal cells were obtained from female WT and PAI-1 KO mice as previously described [20]. Briefly, femur and tibia were removed and subsequently the bone marrow cells were flushed out into Dulbecco's Modified Eagle's Medium (DMEM; Wako) with 10\% FBS. The nonadherent cells were removed by washing using phosphate-buffered saline.

\section{Osteoclast formation}

Bone marrow cells were collected from the femur and tibia of 8-week-old female WT and PAI-1 KO mice. Analysis of osteoclast formation was performed as previously reported [21]. Briefly, bone marrow cells were plated in a 24 -well plate $\left(5.0 \times 10^{5}\right.$ cells/well $)$ and cultured in $\alpha$-MEM with $10 \%$ FBS and $50 \mathrm{ng} / \mathrm{mL} \mathrm{M-CSF}$ for 3 days at $37{ }^{\circ} \mathrm{C}$. Then, osteoclasts were formed in $\alpha$ MEM with $10 \%$ FBS, $50 \mathrm{ng} / \mathrm{mL}$ M-CSF and $50 \mathrm{ng} / \mathrm{mL}$ RANKL for a further 3 days at $37^{\circ} \mathrm{C}$. Detection of osteoclasts was performed using a tartrate-resistant acid phosphatase (TRAP) staining kit (Wako). The numbers of TRAP-positive multinucleated cells (MNCs) were counted in a certain area within the well.

\section{Statistical analysis}

All data were expressed as the mean \pm SEM. Two-way ANOVAs were used to compare the effects of OVX and DMM on both mouse genotypes (WT and PAI-1 KO). When significant differences were observed, individual means were compared using Tukey-Kramer post hoc tests. Statistical values at $P<0.05$ were considered to be significant. All statistical analyses were performed using the Prism software, version 7 (GraphPad Software, Inc., La Jolla, CA, USA).

\section{Results}

Effects of DMM, OVX and PAI-1 deficiency on BMD of subchondral bone in mice

We evaluated the trabecular BMD of the tibial subchondral bone, in which ROI were defined as $240 \mu \mathrm{m}$ (10-slice) segments from $72 \mu \mathrm{m}$ proximal to the end of the proximal growth plate towards the joint using quantitative CT analysis. OVX significantly decreased the trabecular BMD of the subchondral bone in WT and PAI-1 KO mice (Fig. 1a, 

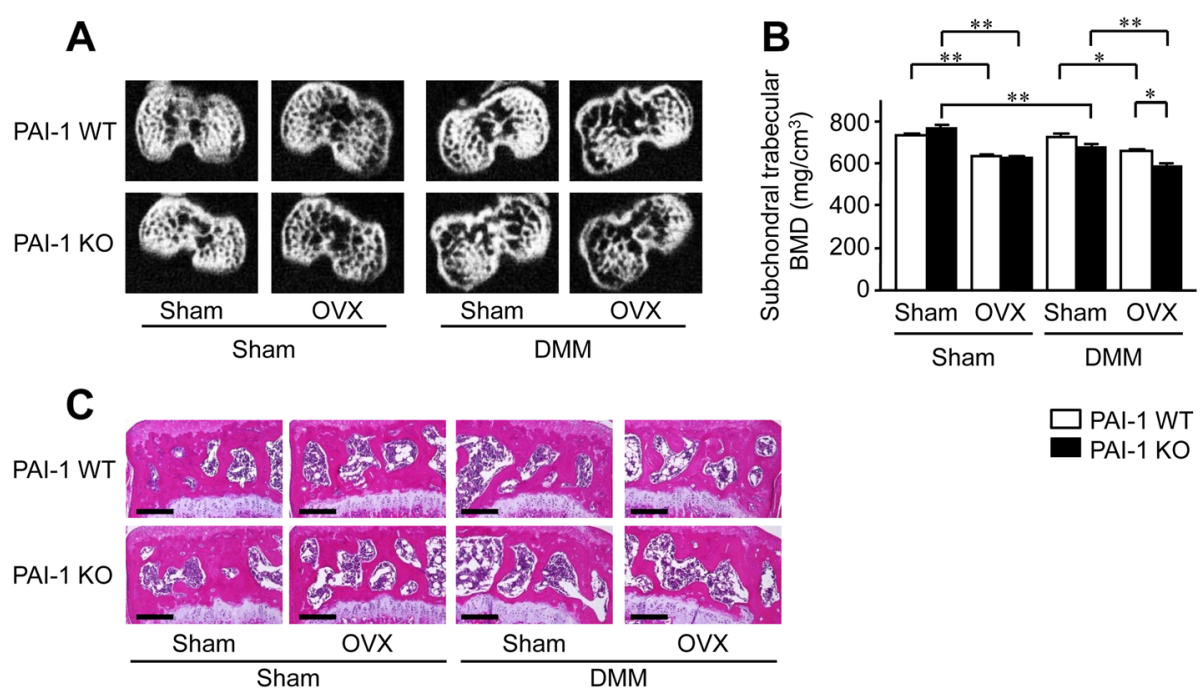

Fig. 1 Effects of DMM, OVX and PAl-1 deficiency on the BMD of the tibial subchondral bone in mice. a qCT images of the subchondral bone in WT and PAI-1 KO mice 8 weeks after DMM or sham surgery with or without OVX. $\mathbf{b}$ The trabecular BMD of the subchondral bone was assessed by qCT in WT and PAI-1 KO mice 8 weeks after DMM or sham surgery with or without OVX. Regions of interest were defined as 240- $\mu \mathrm{m}$ (10-slice) segments from $72 \mu \mathrm{m}$ proximal to the end of the proximal growth plate towards the joint. Data represent the mean \pm SEM of 10-11 mice. ${ }^{* *} p<0.01$ and ${ }^{*} p<0.05$. c Hematoxylin and Eosin-stained sections from the subchondral bone in WT and PAl-1 KO mice 8 weeks after DMM or sham surgery with or without OVX. Scale bars $=200 \mu \mathrm{m}$

b). DMM significantly decreased the trabecular BMD of the subchondral bone in PAI-1 KO mice, although DMM did not affect it in WT mice with or without OVX. PAI-1 deficiency significantly augmented a decrease in the trabecular BMD of the subchondral bone in mice with OVX and DMM (Fig. 1a, b). Histological analysis findings were compatible with those results in qCT (Fig. 1c).

\section{Effects of DMM, OVX and PAI-1 deficiency on articular cartilage degeneration in mice}

Next, we examined the effects of OVX, DMM and PAI-1 deficiency on articular cartilage degeneration in mice. The progression of arthropathy at the knees was evaluated by an OARSI scoring system, which is a widely used method for the evaluation of osteoarthritis. As shown in Fig. 2, there were no significant differences in the OARSI score between WT and PAI-1 KO mice with or without OVX. DMM significantly increased the OARSI score in WT and PAI-1 KO mice, although OVX did not affect the OARSI score. OARSI scores seemed to be elevated in PAI-1 KO mice, compared to those in WT mice, especially in mice with both OVX and DMM, although their differences were not statistically significant.

Roles of IL-1 $\beta$ and PAI-1 deficiency in primary osteoblasts We investigated the mechanisms by which PAI-1 deficiency augments subchondral osteopenia induced by DMM in mice using mouse primary osteoblasts from WT and PAI- 1 KO mice. IL- $1 \beta$ and TGF- $\beta$ are important and representative factors for the pathogenesis of
OA [22]. We therefore examined the roles of IL-1 $\beta$ and TGF- $\beta$ signals in WT and PAI-1 KO mice to clarify the roles of PAI-1 in the pathological state of OA in vitro. Both IL- $1 \beta$ and TGF- $\beta$ significantly enhanced PAI-1 mRNA levels in mouse osteoblasts (Fig. 3a). However, IL-1 $\beta$ did not affect TGF- $\beta$ mRNA levels in these cells from both WT and PAI-1 KO mice (Fig. 3b). Next, we examined the effects of IL-1 $\beta$ and PAI-1 deficiency on osteoblast differentiation in mouse osteoblasts from WT and PAI-1 KO mice. As shown in Fig. 4a, IL-1 $\beta$ significantly decreased Osterix mRNA levels in WT mice,

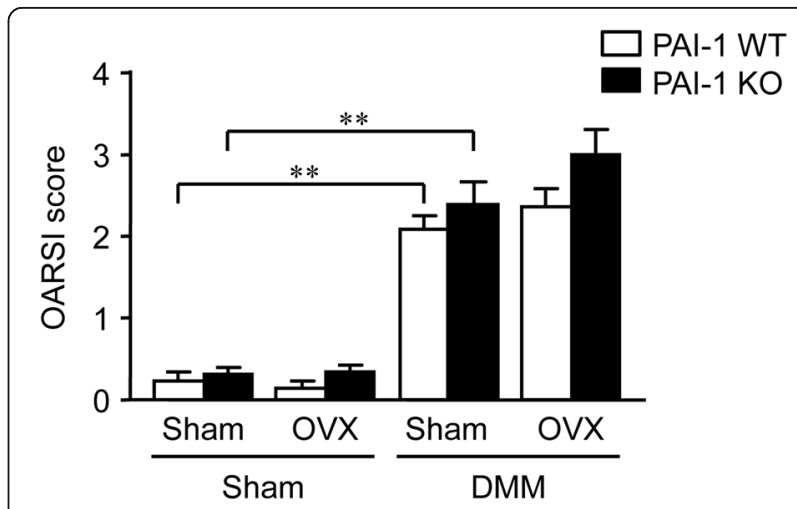

Fig. 2 Effects of DMM, OVX and PAl-1 deficiency on articular cartilage degeneration in mice. Cartilage degeneration was assessed using the OARSI histological scoring system in WT and PAl-1 KO mice 8 weeks after DMM or sham surgery with or without OVX. Each knee was evaluated using 6 grades for the progression of arthropathy. Data represent the mean \pm SEM of $10-11$ mice. ${ }^{* *} p<0.01$ 

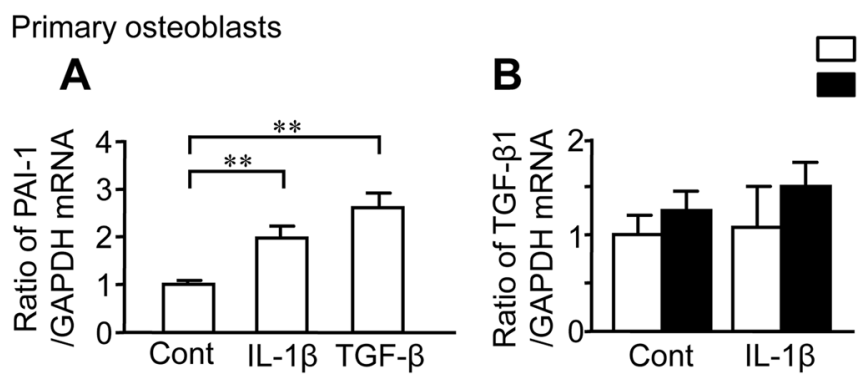

Fig. 3 Effects of IL-1 $\beta$ on the expressions of PAI-1 and TGF- $\beta_{1}$ in primary mouse osteoblasts. a Primary osteoblasts were obtained from 3-day-old

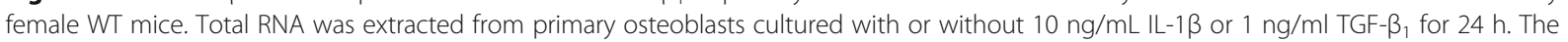
levels of PAI-1 or GAPDH mRNA were assessed by real-time PCR. Data represent the mean \pm SEM. $n=6$ for each group. ${ }^{* *} p<0.01$. b Primary osteoblasts were obtained from 3-day-old female WT and PAI-1 KO mice. Total RNA was extracted from primary osteoblasts cultured with or without $10 \mathrm{ng} / \mathrm{mL} \mathrm{IL}-1 \beta$ for $24 \mathrm{~h}$. Then, the levels of TGF- $\beta_{1}$ or GAPDH mRNA were assessed by real-time PCR. Data represent the mean \pm SEM. $n=6$ for each group

although its effects on other osteogenic genes, such as Runx2, alkaline phosphatase (ALP), type I collagen (Col1 ) and osteocalcin $(\mathrm{OCN})$ were not significant. PAI-1 deficiency did not affect Osterix mRNA levels suppressed by IL-1 $\beta$ (Fig. 4a). Moreover, SB431542, an inhibitor of activin-like kinase (ALK)5, TGF- $\beta$ receptor type I kinase, did not affect Osterix mRNA levels suppressed by IL-1 $\beta$, although it significantly blunted Osterix mRNA levels suppressed by TGF- $\beta$ in osteoblasts (Fig. 4b). We next investigated the involvement of PAI-1 in osteoblast differentiation from mouse bone marrow cells in the presence or absence of IL-1 $\beta$. As shown in Fig. 4c, PAI-1 deficiency did not affect the mRNA levels of Osterix, ALP and OCN in bone marrow cells treated with
BMP-2 in the presence or absence of IL-1 $\beta$, suggesting that PAI-1 deficiency does not affect osteoblastic differentiation from mouse bone marrow cells.

\section{Effects of PAl-1 deficiency on osteoclast formation in mice}

Finally, we investigated the effects of PAI-1 deficiency on osteoclast formation in WT and PAI-1 KO mice. RANKL is a crucial factor for osteoclast formation and activity, and osteoprotegerin (OPG) inhibits osteoclast formation by binding to RANKL. RANKL and OPG are the chief regulators of bone resorption. As shown in Fig. 5, IL-1 $\beta$ significantly elevated RANKL mRNA levels as well as the ratio of RANKL/OPG mRNA in

\section{A}
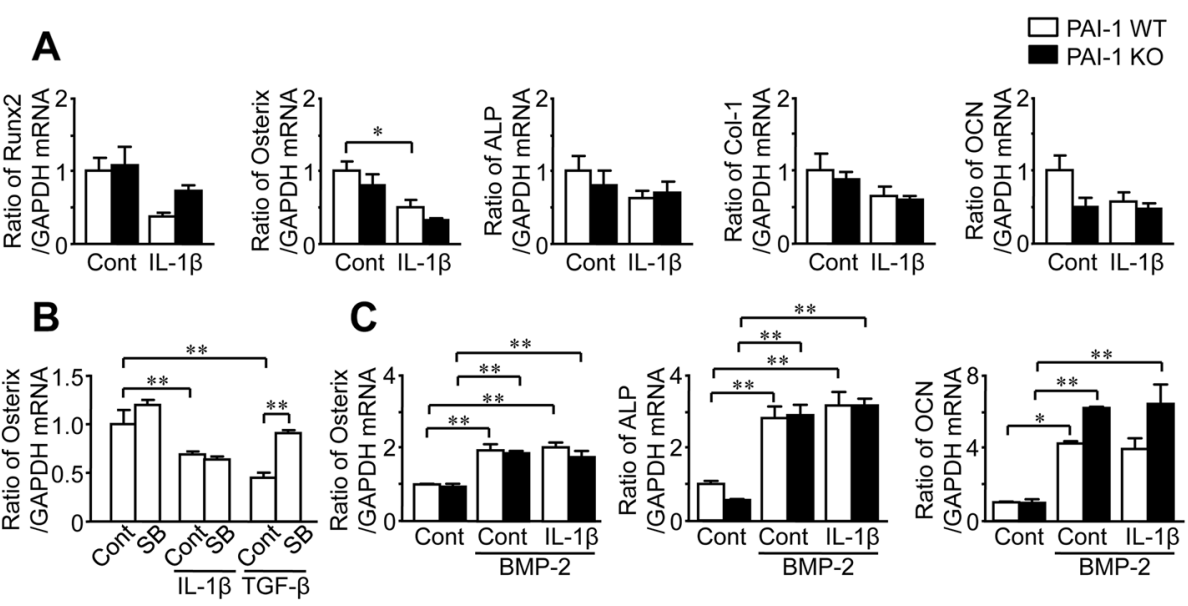

C
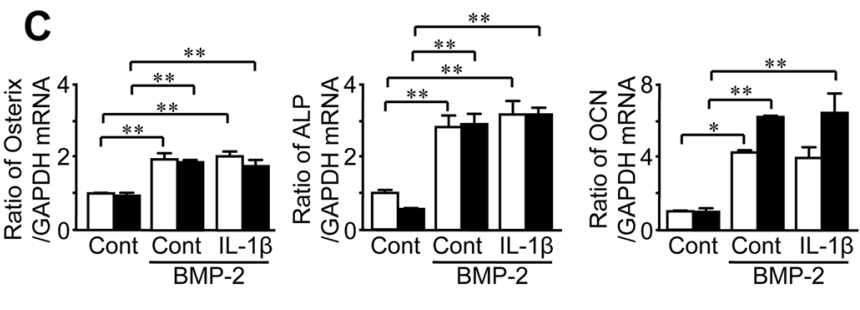

Fig. 4 Effects of PAl-1 deficiency on osteoblast differentiation in mouse osteoblasts and bone marrow cells. a Primary osteoblasts were obtained from 3-day-old female WT and PAl-1 KO mice. Total RNA was extracted from primary osteoblasts cultured with or without $10 \mathrm{ng} / \mathrm{mL} I \mathrm{~L}-1 \beta$ or $1 \mathrm{ng} / \mathrm{ml}$ TGF- $\beta_{1}$ for $24 \mathrm{~h}$. The levels of Runx2, Osterix, ALP, Col-1, OCN or GAPDH mRNA were assessed by real-time PCR. Data represent the mean \pm SEM. $n=6$ for each group. ${ }^{*} p<0.05$. b Primary osteoblasts were obtained from 3-day-old female WT mice. Total RNA was extracted from primary osteoblasts cultured with or without $10 \mathrm{ng} / \mathrm{mL}$ IL-1 $\beta$ or $1 \mathrm{ng} / \mathrm{ml} \mathrm{TGF}-\beta_{1}$ in the presence or absence of $1 \mu \mathrm{M}$ SB421542 for $24 \mathrm{~h}$. The levels of Osterix or GAPDH mRNA were assessed by real-time PCR. Data represent the mean \pm SEM. $n=6$ for each group. ${ }^{* *} p<0.01$. c Bone marrow cells were obtained from 8-week-old female WT and PAl-1 KO mice. Total RNA was extracted from the bone marrow cells cultured with or without $200 \mathrm{ng} / \mathrm{ml}$ BMP-2 or $10 \mathrm{ng} / \mathrm{mL}$ IL-1 $\beta$ for $48 \mathrm{~h}$. The levels of Osterix, ALP, OCN or GAPDH mRNA were assessed by real-time PCR. Data represent the mean \pm SEM. $n=5$ for each group. ${ }^{* *} p<0.01$ and ${ }^{*} p<0.05$ 


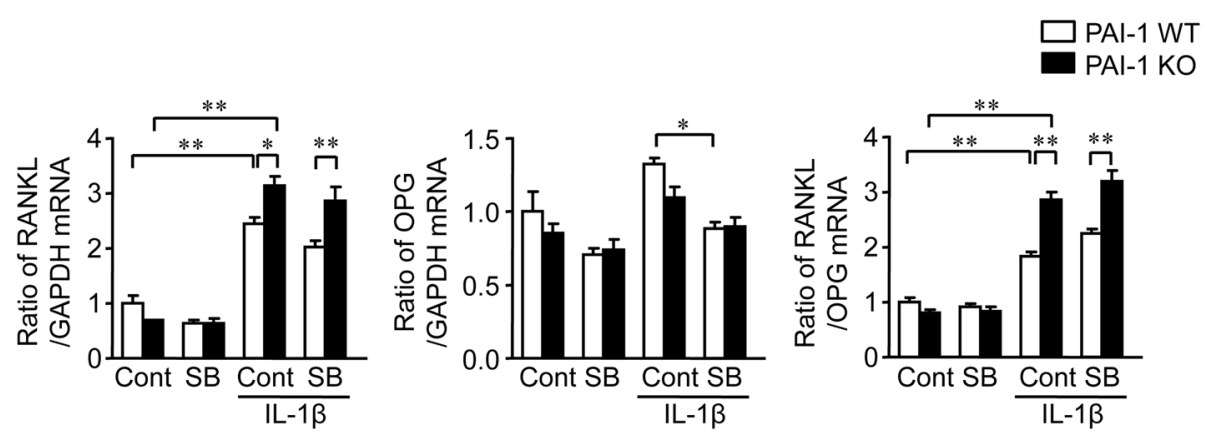

Fig. 5 Effects of PAI-1 deficiency on the expression of RANKL and OPG in mouse osteoblasts. Primary osteoblasts were obtained from 3-day-old female WT and PAl-1 KO mice. Total RNA was extracted from primary osteoblasts cultured with or without $10 \mathrm{ng} / \mathrm{mL} / \mathrm{L}-1 \beta$ or $1 \mathrm{ng} / \mathrm{ml} \mathrm{TGF}-\beta_{1}$ in the presence or absence of $1 \mu \mathrm{M}$ SB421542 for $24 \mathrm{~h}$. The levels of RANKL, OPG or GAPDH mRNA were assessed by real-time PCR. Data represent the mean \pm SEM. $n=6$ for each group. ${ }^{* *} p<0.01$ and ${ }^{*} p<0.05$

osteoblasts from WT mice. PAI-1 deficiency significantly augmented this IL-1ß-enhanced RANKL mRNA level and RANKL/OPG mRNA ratio in osteoblasts. However, SB431542, a TGF- $\beta$ signaling inhibitor, did not affect the IL-1 $\beta$-enhanced RANKL mRNA level and RANKL/OPG mRNA ratio in osteoblasts from mice with or without PAI-1 deficiency.

We examined the effects of PAI-1 deficiency of osteoclast formation from mouse bone marrow cells obtained from WT and PAI-1 KO mice. PAI-1 deficiency significantly increased the number of TRAP-positive MNCs in the presence or absence of IL-1 $\beta$ in mouse bone marrow cell cultures in the presence of M-CSF and RANKL (Fig. 6).

\section{Discussion}

In the present study, we showed that PAI-1 deficiency augmented subchondral osteopenia after induction of OA and/or OVX in mice. PAI-1 deficiency enhanced

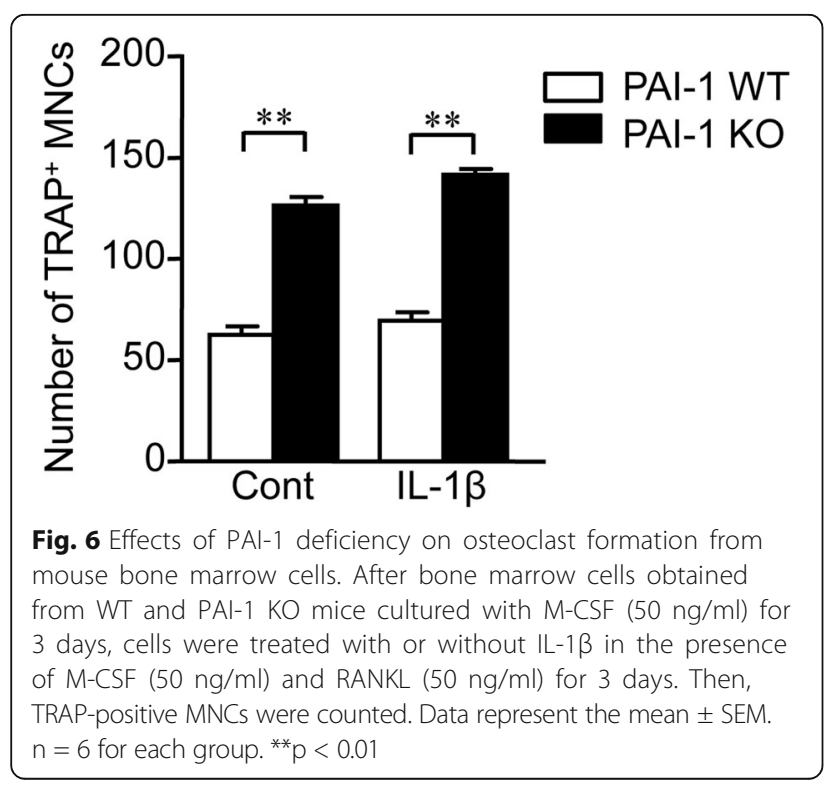

osteoclast formation from mouse bone marrow cells as well as RANKL expression induced by IL-1 $\beta$ in mouse osteoblasts, although it did not affect osteoblast differentiation suppressed by IL-1 $\beta$.

Subchondral osteopenia as well as the destruction of joint cartilage are important for the pathogenesis of OA $[23,24]$. The initial change of cartilage induced by excessive mechanical stress in OA may cause the changes to the subchondral bone through inflammatory cytokines, such as IL-1 $\beta$ [25]. On the other hand, the initial change of the subchondral bone and subsequent local cytokine release might induce cartilage degeneration. The contribution of both mechanisms in the pathogenesis of OA is still controversial. In the present study, DMM significantly enhanced subchondral bone osteopenia in PAI-1-deficient mice with or without OVX, suggesting that knee OA progression induces subchondral osteopenia at least PAI-1 deficient state in mice. We speculate that the trabecular BMD decrease in the subchondral bone represents local subchondral osteopenia after induction of OA in this study. However, further analysis using micro $\mathrm{CT}$ with higher resolution will be necessary for the detection of concrete subchondral bone changes in mice.

We and others have previously reported the pathophysiological roles of PAI-1 in bone and cartilage [13-15]. Rundle et al. reported that the fracture callus was enlarged in PAI-1-deficient mice [26]. Moreover, we showed that circulating PAI-1 might be involved in diabetic osteopenia and delayed bone repair as well as glucocorticoid-induced osteoporosis in mice [13-15]. In that study, exogenous addition of PAI-1 suppresses osteoblast differentiation in mouse osteoblasts [13]. In contrast, in the present study, PAI-1 deficiency significantly augmented subchondral osteopenia after induction of $\mathrm{OA}$ with or without OVX in mice. These findings suggest that PAI-1 might protect against subchondral osteopenia after induction of $\mathrm{OA}$ in mice. 
IL-1 $\beta$ plays central roles as an inflammatory cytokine in the pathogenesis of OA [27]. IL-1 $\beta$ induces bone resorption, which is related to subchondral osteopenia in OA. In the present study, PAI-1 deficiency did not affect genes related to osteoblast differentiation that are suppressed by IL-1 $\beta$ in mouse osteoblasts, although IL-1 $\beta$ significantly enhanced the expression of PAI-1. On the other hand, PAI-1 deficiency significantly enhanced osteoclast formation from mouse bone marrow cells in the presence of $\mathrm{M}$ CSF and RANKL. Moreover, PAI-1 deficiency significantly augmented the expression of RANKL and the ratio of RANKL/OPG mRNA in mouse primary osteoblasts. These findings suggest that PAI-1 protects against bone resorption in the subchondral bone after induction of OA, although PAI-1 does not affect osteoblast differentiation suppressed after induction of OA. This finding might partly explain the mechanisms by which PAI-1 deficiency augments subchondral osteopenia in mice. Several studies indicate that increases in osteoclast number and bone resorption are observed at the subchondral bone in patients or animals with OA [23, 24, 28-30]. Moreover, PAI-1 deficiency promoted bone remodeling during fracture healing in mice [26]. Taken together, PAI-1 might suppress the enhancement of bone resorption and subsequent subchondral osteopenia after induction of OA in mice. Subchondral osteopenia enhanced by PAI- 1 deficiency seemed to augment cartilage degeneration, although the statistical differences were not significant in the present study.

Previous studies indicate that TGF- $\beta$ is involved in the pathogenesis of OA $[28,31]$. Moreover, TGF- $\beta$ is related to subchondral osteopenia after induction of OA [28]. In the present study, inhibition of TGF- $\beta$ signaling by an ALK5 inhibitor did not affect the effects of IL-1 $\beta$ on osteoblast differentiation and RANKL expression in mouse osteoblasts. Moreover, IL- $1 \beta$ did not affect TGF- $\beta$ expression in osteoblasts from both WT and PAI-1 KO mice. These findings indicate that the effects of PAI-1 deficiency on the enhancement of osteoclastic bone resorption are independent from TGF- $\beta$ signaling in mice.

Postmenopausal osteoporosis may be a risk factor for the development of knee OA [32]. Several studies suggest that $\mathrm{OA}$ is accelerated in postmenopausal women $[3,4]$. The influence of OVX on OA progression in animal studies is still controversial [33, 34], although Miyatake et al. reported that OVX exacerbates the severity of OA induced by treadmill exercise in mice [35]. Our data showed that OVX significantly enhances subchondral osteopenia induced by DMM in PAI-1-deficient mice, but not in WT mice. From these findings, we speculate that OVX might enhance subchondral osteopenia when the suppression of PAI-1 on osteoclast formation was cancelled. There were neither additive nor synergistic effects in the influences of OVX and DMM on the subchondral trabecular BMD in the present study. Although the reason remains unknown, IL-1 $\beta$ has been known to be involved in the pathogenesis of osteopenia induced by both estrogen deficiency and OA [27, 36] .

Our study suggested that an induction of local endogenous PAI-1 at the subchondral bone suppresses subchondral osteopenia after induction of $\mathrm{OA}$ in mice. Clinical evidence indicates that PAI-1 is related to the pathophysiology of various diseases, such as diabetes, cardiovascular diseases, glucocorticoid excess and cancer, as a malignant adipocytokine [12]. In contrast, the protective role of PAI-1 of the subchondral bone in the pathophysiology of knee OA is contrary to the action of PAI-1 as a malignant adipocytokine [12]. Further studies are necessary to clarify the roles of PAI-1 in tissues such as the cartilage and synovial cells to investigate the detailed roles of PAI-1 in the pathogenesis of OA.

\section{Conclusions}

In conclusion, we demonstrated that PAI-1 deficiency enhances subchondral osteopenia after induction of OA in mice. An enhancement of IL-1 $\beta$-induced RANKL expression and subsequent osteoclast formation by PAI-1 deficiency might be related to this mechanism.

\begin{abstract}
Abbreviations
ALK: Activin-like kinase; ALP: Alkaline phosphatase; BMD: Bone mineral density; BMP-2: Bone morphogenetic protein-2; Col-1: Type I collagen; DMEM: Dulbecco's modified eagle's medium; DMM: Destabilization of the medial meniscus; FBS: Fetal bovine serum; GAPDH: Glyceraldehyde 3phosphate dehydrogenase; IL-1 $\beta$ : Interleukin-1 $\beta$; M-CSF: Macrophage colonystimulating factor; MNCs: Multinucleated cells; OA: Osteoarthritis; OARSI: Osteoarthritis research society international; OCN: Osteocalcin; OPG: Osteoprotegerin; OVX: Ovariectomy; PAl-1 KO: Plasminogen activator inhibitor-1-deficient; PAl-1: Plasminogen activator inhibitor-1;

QCT: Quantitative computed tomography; RANKL: Receptor activator of nuclear factor-KB ligand; ROI: Regions of interest; TGF- $\beta_{1}$ : Transforming growth factor- $\beta_{1}$; TRAP: Tartrate-resistant acid phosphatase; WT: Wild type; $a-$ MEM: Minimum essential medium alpha modification
\end{abstract}

\section{Acknowledgments}

The authors acknowledge the members of the Life Science Research Institute, Kindai University for their technical support and advice.

\section{Funding}

This study was partly supported by a grant from the Takeda Science Foundation to N. K. and a Grant-in-Aid for Scientific Research C: 16 K08534 to N. K., 15 K08220 to H. K. and a Grant-in-Aid for Scientific Research on Innovative Areas (grant number 15H05935, "Living in Space") from the Ministry of Education, Culture, Sports, Science, and Technology of Japan.

\section{Availability of data and materials}

The data sets used for the current study are available from the corresponding authors upon reasonable request.

\section{Authors' contributions}

$\mathrm{AM}, \mathrm{NK}, \mathrm{MA}$ and $\mathrm{HK}$ contributed to the conception and design of the research. AM, NK, KOka, KT, MI and KOku performed the experiments. AM and $\mathrm{NK}$ analyzed the data. $\mathrm{AM}, \mathrm{NK}, \mathrm{OM}, \mathrm{MA}$ and $\mathrm{HK}$ interpreted the results of the experiments. AM, NK and HK prepared the figs. AM and HK drafted the manuscript. OM, MA and HK edited and revised the manuscript. All authors approved final version of manuscript. 


\section{Ethics approval}

All animal experiments were approved by the Committee for the Care and Use of Laboratory Animals at Kindai University (KAME-28-19). All procedures for animal experiments were performed according to the guidelines of the National Institutes of Health and the institutional rules for the use and care of laboratory animals at Kindai University.

\section{Consent for publication}

Not applicable

\section{Competing interests}

The authors declare there are no competing interests.

\section{Publisher's Note}

Springer Nature remains neutral with regard to jurisdictional claims in published maps and institutional affiliations.

\section{Author details}

'Department of Orthopaedic Surgery, Kindai University Faculty of Medicine, Osakasayama, Japan. ${ }^{2}$ Department of Physiology and Regenerative Medicine, Kindai University Faculty of Medicine, 377-2 Ohnohigashi, Osakasayama, Osaka 589-8511, Japan. ${ }^{3}$ Life Science Research Institute, Kindai University, Osakasayama, Japan

Received: 26 May 2017 Accepted: 5 September 2017

\section{Published online: 11 September 2017}

\section{References}

1. Rangger C, Kathrein A, Klestil T, Glötzer W. Partial meniscectomy and osteoarthritis. Implications for treatment of athletes. Sports Med. 1997:23: 61-8.

2. Srikanth VK, Fryer JL, Zhai G, Winzenberg TM, Hosmer D, Jones G. A metaanalysis of sex differences prevalence, incidence and severity of osteoarthritis. Osteoarthritis Cartilge. 2005:13:769-81.

3. Helmick CG, Felson DT, Lawrence RC, Gabriel S, Hirsch R, Kwoh CK, et al. Estimates of the prevalence of arthritis and other rheumatic conditions in the United States. Part I. Arthritis Rheum. 2008:58:15-25.

4. Roman-Blas JA, Castañeda S, Largo R, Herrero-Beaumont G. Osteoarthritis associated with estrogen deficiency. Arthritis Res Ther. 2009;11:241.

5. Wang CJ, Huang CY, Hsu SL, Chen JH, Cheng JH. Extracorporeal shockwave therapy in osteoporotic osteoarthritis of the knee in rats; an experiment in animals. Arthritis Res Ther. 2014;16:R139.

6. Radin EL, Rose RM. Role of subchondral bone in the initiation and progression of cartilage damage. Clin Orthop Relate Res. 1986;213:34-40.

7. Burr DB. The importance of subchondral bone in osteoarthrosis. Curr Opin Rheumatol. 1998;10:256-62

8. Pastoureau P, Leduc S, Chomel A, De Ceuninck F Quantitative assessment of articular cartilage and subchondral bone histology in the meniscectomized guinea pig model of osteoarthritis. Osteoarthr Cartil. 2003; 11:412-23.

9. Hayami T, Pickarski M, Wesolowski GA, McLane J, Bone A, Destefano J, et al. The role of subchondral bone remodeling in osteoarthritis: reduction of cartilage degeneration and prevention of osteophyte formation by alendronate in the rat anterior cruciate ligament transection model. Arthritis Rheum. 2004;50:1193-206.

10. Buckland-Wright JC, Messent EA, Bingham CO 3rd, Ward RJ, Tonkin C. A 2yr longitudinal radiographic study examining the effect of a bisphosphonate (risedronate) upon subchondral bone loss in osteoarthritic knee patients. Rheumatology (Oxford). 2007:46:257-64.

11. Conde J, Scotece M, Gómez R, López V, Gómez-Reino JJ, Lago F, et al. Adipokines: biofactors from white adipose tissue. A complex hub among inflammation, metabolism, and immunity. Biofactors. 2011;37:413-20.

12. Kaji H. Adipose tissue-derived plasminogen activator inhibitor-1: function and regulation. Compr Physiol. 2016;6:1873-96.

13. Tamura Y, Kawao N, Okada K, Yano M, Okumoto K, Matsuo O, et al. Plasminogen activator inhibitor-1 is involved in streptozotocin-induced bone loss in female mice. Diabetes. 2013:62:3170-9.

14. Mao L, Kawao N, Tamura Y, Okumoto K, Okada K, Yano M, et al. Plasminogen activator inhibitor-1 is involved in impaired bone repair associated with diabetes in female mice. PLoS One. 2014;9:e92686.
15. Tamura Y, Kawao N, Yano M, Okada K, Okumoto K, Chiba Y, et al. Role of plasminogen activator inhibitor-1 in glucocorticoid-induced diabetes and osteopenia in mice. Diabetes. 2015;64:2194-206.

16. Shiomi A, Kawao N, Yano M, Okada K, Tamura Y, Okumoto K, et al. a2Antiplasmin is involved in bone loss induced by ovariectomy in mice. Bone. 2015;79:233-41.

17. Glasson SS, Blanchet TJ, Morris EA. The surgical destabilization of the medial meniscus (DMM) model of osteoarthritis in the 129/SvEv mouse. Osteoarthr Cartil. 2007:15:1061-9.

18. Tamura Y, Kawao N, Yano M, Okada K, Matsuo O, Kaji H. Plasminogen activator inhibitor-1 deficiency ameliorates insulin resistance and hyperlipidemia but not bone loss in obese female mice. Endocrinology. 2014:155:1708-17.

19. Glasson SS, Chambers MG, Van Den Berg WB, Little CB. The OARSI histopathology initiative - recommendations for histological assessments of osteoarthritis in the mouse. Osteoarthr Cartil. 2010;18(Suppl 3):S17-23.

20. Kawao N, Tamura Y, Okumoto K, Yano M, Okada K, Matsuo O, et al. Plasminogen plays a crucial role in bone repair. J Bone Miner Res. 2013;28: 1561-74.

21. Saito E, Suzuki D, Kurotaki D, Mochizuki A, Manome Y, Suzawa T, et al. Down-regulation of Iff8 by Lyz2-cre/loxP accelerates osteoclast differentiation in vitro. Cytotechnology. 2017:69:443-50.

22. Yuan XL, Meng HY, Wang YC, Peng J, Guo QY, Wang AY, et al. Bonecartilage interface crosstalk in osteoarthritis: potential pathways and future therapeutic strategies. Osteoarthr Cartil. 2014;22:1077-89.

23. Suri S, Walsh DA. Osteochondral alterations in osteoarthritis. Bone. 2012:51: 204-11

24. Li G, Yin J, Gao J, Cheng TS, Pavlos NJ, Zhang C, et al. Subchondral bone in osteoarthritis: insight into risk factors and microstructural changes. Arthritis Res Ther. 2013;15:223.

25. Mastbergen SC, Lafeber FP. Changes in subchondral bone early in the development of osteoarthritis. Arthritis Rheum. 2011;63:2561-3.

26. Rundle $\mathrm{CH}$, Wang $\mathrm{X}$, Wergedal JE, Mohan S, Lan KH. Fracture healing in mice deficient in plasminogen activator inhibitor-1. Calcif Tissue Int. 2008;83: 276-84

27. Pujol JP, Chadjichristos C, Legendre F, Bauge C, Beauchef $G$, Andriamanalijaona $\mathrm{R}$, et al. Interleukin-1 and transforming growth factorbeta 1 as crucial factors in osteoarthritic cartilage metabolism. Connect Tissue Res. 2008:49:293-7.

28. Ochi S, Shinohara M, Sato K, Gober HJ, Koga T, Kodama T, et al. Pathological role of osteoclast costimulation in arthritis-induced bone loss. Proc Nat Acad Sci U S A. 2007:104:11394-9.

29. Botter SM, van Osch GJ, Clockaerts S, Waarsing JH, Weinans $H$, van Leeuwen IP. Osteoarthritis induction leads to early and temporal subchondral plate porosity in the tibial plateau of mice: an in vivo microfocal computed tomography study. Arthritis Rheum. 2011:63:2690-9.

30. Bertuglia A, Lacourt M, Girard C, Beauchamp G, Richard H, Laverty S. Osteoclasts are recruited to the subchondral bone in naturally occurring post-traumatic equine carpal osteoarthritis and may contribute to cartilage degradation. Osteoarthr Cartil. 2016:24:555-66.

31. Zhen G, Cao X. Targeting TGF- $\beta$ signaling in subchondral bone and articular cartilage homeostasis. Trends Pharmacol Sci. 2014;35:227-36.

32. Roman-Blas JA, Castañeda S, Largo R, Lems WF, Herrero-Beaumont G. An OA phenotype may obtain major benefit from bone-acting agents. In Seminars Arthritis Rheumatism. 2014:43:421-8.

33. Sniekers $Y H$, Weinans $H$, Bierma-Zeinstra SM, van Leeuwen JP, van Osch GJ. Animal models for osteoarthritis: the effect of ovariectomy and estrogen treatment - a systematic approach. Osteoarthr Cartil. 2008;16:533-41.

34. de Klerk BM, Schiphof D, Groeneveld FP, Koes BW, van Osch GJ, van Meurs $J B$, et al. No clear association between female hormonal aspects and osteoarthritis of the hand, hip and knee: a systematic review. Rheumatology (Oxford). 2009:48:1160-5.

35. Miyatake K, Muneta T, Ojima M, Yamada J, Matsukura Y, Abula K, et al. Coordinate and synergistic effects of extensive treadmill exercise and ovariectomy on articular cartilage degeneration. BMC Musculoskelet Disord. 2016:17.238.

36. Brincat SD, Borg M, Camilleri G, Calleja-Agius J. The role of cytokines in postmenopausal osteoporosis. Minerva Ginecol. 2014;66:391-407. 